\title{
A espessura do músculo adutor do polegar não está associada a Proteína C Reativa em pacientes com câncer hospitalizados
}

\author{
Adductor pollicis muscle thickness is not associated with C-reactive protein in hospitalized \\ cancer patients
}

DOI: $10.37111 /$ braspenj.2020354003

Nathália da Cunha Ferrél

Rebeca Palhares Barbosa

Thaís Cristina Borges ${ }^{2}$

Gustavo Duarte Pimentel ${ }^{3}$

\section{Unitermos:}

Neoplasias. Inflamação. Músculos. Hospitalização.

\section{Keywords:}

Neoplasms. Inflammation. Muscles. Hospitalization.

\section{Endereço para correspondência:}

Gustavo Duarte Pimentel

Faculdade de Nutrição, Universidade Federal de Goiás

Rua 227, Quadra 68, s/ $\mathrm{n}^{\circ}$ - Setor Leste Universitário

- Goiânia, GO, Brasil - CEP 74605-080

E-mail: gupimentel@yahoo.com.br

\section{Submissão}

1 de julho de 2020

Aceito para publicação

12 de dezembro de 2020

\begin{abstract}
RESUMO
Objetivo: Avaliar a associação entre as concentrações sanguíneas de proteína $C$ reativa com um indicador de massa muscular em pacientes oncológicos hospitalizados. Método: Estudo observacional analítico do tipo transversal, realizado com 110 indivíduos de ambos os sexos, $\geq 18$ anos, com câncer sólido e/ou líquido e em tratamento clínico e/ou cirúrgico. Os dados clínicos e socioeconômicos foram obtidos de prontuários médicos. A espessura do músculo adutor do polegar foi obtida com o adipômetro. Os pacientes foram divididos em dois grupos, baixa espessura muscular $(<13,4 \mathrm{~mm})$ e espessura normal $(\geq 13,4 \mathrm{~mm})$. A regressão logística foi realizada para verificar a associação entre a espessura do músculo adutor do polegar e a proteína $\mathrm{C}$ reativa. Resultados: Observou-se que $90 \%$ ( $n=100)$ dos pacientes foram classificados com baixa espessura muscular, maior prevalência de câncer sólido, menor peso usual, peso atual e índice de massa corporal. A análise de regressão logística não revelou associação, tanto no modelo sem ajuste (OR: 1,01; IC95\% [0,94-1,09], $p=0,65$ ), quanto no modelo ajustado para sexo e idade (OR: 1,01; IC95\% $[0,94-1,09], p=0,61)$ e para sexo, idade e índice de massa corporal (IMC) (OR: 1,04; IC95\% $[0,96-1,13], p=0,29)$. Conclusão: Embora a espessura do músculo adutor do polegar seja um método validado para avaliação nutricional, não foi observada associação com o marcador de inflamação (proteína $\mathrm{C}$ reativa).
\end{abstract}

\section{ABSTRACT}

Objective: To evaluate the association between blood of $\mathrm{C}$-reactive protein concentrations and an indicator of muscle mass in hospitalized cancer patients. Methods: A cross-sectional study carried out with 110 patients of both sexes, aged $\geq 18$ years, with solid and/or liquid cancer undergoing clinical and/or surgical treatment. Clinical and socioeconomic data were obtained from medical records. The adductor pollicis muscle thickness was obtained using skinfold caliper. Patients were divided into two groups: altered adductor pollicis muscle thickness $(<13.4 \mathrm{~mm})$ and normal adductor pollicis muscle thickness ( $\geq 13.4 \mathrm{~mm}$ ). Logistic regression was performed to verify the association between adductor pollicis muscle thickness and C-reactive protein. Results: It was observed that $90 \%(n=100)$ of the patients were classified with reduced adductor pollicis muscle thickness, higher prevalence of the solid tumor, low usual weight, current weight, and body mass index. The logistic regression analysis showed no association between the adductor pollicis muscle thickness and $\mathrm{C}$-reactive protein concentrations, in any of models used: model without adjustment (OR: 1.01; $95 \% \mathrm{Cl}$ [0.94-1.09], $\mathrm{p}=0.65$ ); adjusted for sex and age (OR: 1.01; $95 \% \mathrm{Cl}[0.94-1.09], \mathrm{p}=0.61)$; and adjusted model for sex, age and body mass index (BMI) (OR: $1.04 ; 95 \% \mathrm{Cl}[0.96-1.13], \mathrm{p}=0.29)$. Conclusion: Although adductor pollicis muscle thickness is validated method for nutritional assessment, we did not find an association with the inflammation marker (C-reactive protein).

1 Nutricionista pela Universidade Federal de Goiás (UFG). Laboratório de Pesquisa em Nutrição Clínica e Esportes (Labince), Faculdade de Nutrição, Universidade Federal de Goiás, Goiânia, GO, Brasil.

2 Nutricionista e Mestre em Nutrição pela UFG. Laboratório de Pesquisa em Nutrição Clínica e Esportes (Labince), Faculdade de Nutrição, Universidade Federal de Goiás, Goiânia, GO, Brasil.

3 Nutricionista, Doutor pela Universidade Estadual de Campinas (UNICAMP). Laboratório de Pesquisa em Nutrição Clínica e Esportes (Labince), Faculdade de Nutrição, Universidade Federal de Goiás, Goiânia, GO, Brasil. 


\section{INTRODUÇ̃̃O}

O câncer é considerado um problema de saúde pública mundial, apresentando altas taxas de incidência, tornandose responsável por aproximadamente $12 \%$ das causas de morte no mundo ${ }^{1,2}$.

Atualmente, estima-se 18,1 milhões de novos casos e 9,6 milhões de mortes por câncer em todo o mundo. Os cânceres de pulmão, mama, colorretal e próstata estão relacionados ao aumento da mortalidade neste grupo. Dentre os tipos de câncer mais incidentes no sexo masculino estão os de próstata, pulmão, colorretal, seguido pelo câncer de estômago e fígado. Já no sexo feminino são os tumores de mama, colo do útero, cólon, reto e pulmão ${ }^{3}$.

O câncer é caracterizado por um conjunto de doenças, que têm em comum o crescimento desordenado das células invadindo tecidos e órgãos, podendo apresentar um potencial metastático ${ }^{4}$. Dentre os problemas metabólicos induzidos pelo câncer, há uma reação inflamatória que se caracteriza por uma resposta de fase aguda, na qual ocorre a liberação de citocinas pró-inflamatórios, tais como IL-1 $\beta$, TNF- $\alpha$, IL-6, dentre outros. Essas citocinas promovem alterações nas concentrações plasmáticas de inúmeras proteínas, aumentando, assim, a expressão de proteínas de fase aguda, tal como a proteína $\mathrm{C}$ reativa $(\mathrm{PCR})^{5}$.

A PCR é um reagente que reflete um dano tecidual, sendo um marcador sensível de inflamação. Altos níveis revelam a intensidade de vários processos patológicos. A PCR atua como indicador prognóstico em locais primários específicos e em doenças avançadas ${ }^{6}$. Valores de PCR acima de 0,5 indicam aumentado estágio inflamatório ${ }^{7}$. Análises prévias demonstraram que, em pacientes com cânceres sólidos, altas concentrações de PCR estão associadas a um pior prognóstico, enquanto que, em indivíduos aparentemente saudáveis, os níveis elevados de PCR estão associados a maior risco para desenvolver câncer ${ }^{8}$.

Em processos inflamatórios, as concentrações séricas são alteradas rapidamente, de forma a auxiliar no diagnóstico precoce do aumento do quadro inflamatório. Nos dias de hoje, temos um método utilizado para mensurar a quantidade de PCR, o qual é chamado de ultrassensível (US), este consegue detectar concentrações menores quando comparado ao exame de PCR normal'.

Embora haja poucos estudos realizados com pacientes oncológicos, os resultados apontam, até o momento, para a eficácia na utilização da medida da espessura do músculo adutor do polegar (EMAP) na avaliação de quadros de desnutrição. Segundo Bragagnolo et al. ${ }^{10}$, a avaliação da massa muscular de pacientes hospitalizados pode ser realizada por meio de uma técnica fácil de ser executada, de forma simples e rápida. Assim, a EMAP vem sendo um método preciso frente aos parâmetros antropométricos.
Ainda que, em pacientes oncológicos em cuidados paliativos exclusivos, a EMAP não foi associada à avaliação subjetiva global produzida pelo próprio paciente (ASGPPP) e à força da preensão manual (FPM), outros estudos observaram que a EMAP se associou com a redução da mortalidade em 30 dias. Dessa forma, os pacientes que vieram a óbito também apresentaram menores valores da EMAP, indicando que essa medida pode auxiliar na melhor intervenção nutricional dos pacientes ${ }^{11,12}$.

Sendo assim, este estudo teve como objetivo verificar a associação da EMAP com a PCR em pacientes oncológicos.

\section{MÉTODO}

\section{Delineamento e População do Estudo}

Estudo observacional analítico do tipo transversal realizado com 110 indivíduos, desses 55 homens e 55 mulheres, internados nas clínicas médica e cirúrgica do Hospital das Clínicas do Hospital das Clínicas da Universidade Federal de Goiás que concordaram voluntariamente em participar.

Os critérios de inclusão adotados foram pacientes de ambos os sexos, com qualquer tipo de câncer, idade $\geq 18$ anos e que estivessem em tratamento clínico e/ou cirúrgico. Esse estudo foi aprovado pelo Comitê de Ética em Pesquisa, sob o protocolo 2.038.651.

\section{Avaliação Clínica e Antropométrica}

Os dados clínicos foram obtidos a partir da aplicação de um questionário padronizado e por meio da análise de prontuário. $\bigcirc$ questionário padronizado contempla: nome do paciente, sexo, leito de internação, número do prontuário, diagnóstico principal, data de nascimento, idade (em anos), data de admissão hospitalar, data de atendimento, se houve perda de peso nos últimos 6 meses, porcentagem de perda de peso, peso usual, peso atual, altura, IMC, PCR e EMAP.

A massa corporal atual $(\mathrm{kg})$ foi obtida utilizando balança calibrada $\left(\right.$ Welmy $\left.{ }^{\circledR}\right)$, com capacidade máxima de $300 \mathrm{~kg}$, instalada em local plano. A altura $(\mathrm{m})$ foi medida no estadiômetro presente na balança, com fração de $0,5 \mathrm{~cm}$ para altura e extensão máxima de 2 metros. Para realização da medida, os pacientes foram posicionados no equipamento, descalços, de forma que a cabeça, os ombros, o quadril e a panturrilha ficassem paralelos à superfície vertical do mesmo, com braços estendidos ao longo do corpo, calcanhares juntos e cabeça formando um ângulo de $90^{\circ} \mathrm{com}$ a base superior do estadiômetro. Tais medidas estão de acordo com a descrição de procedimentos de Heyward e Stolarczyk ${ }^{13}$. Posteriormente, o índice de massa corporal (IMC, $\mathrm{kg} / \mathrm{m}^{2}$ ) foi calculado. 
A aferição da EMAP ocorreu por meio de duas avaliadoras devidamente treinadas, em que os pacientes eram posicionados de forma que os mesmos ficassem sentados com a mão dominante sobre o joelho e o cotovelo em posição de noventa graus ${ }^{14}$. Utilizou-se 0 adipômetro de Lange Skinfold Califper ${ }^{\circledR}$, exercendo uma pressão contínua de $10 \mathrm{~g} / \mathrm{mm}^{2}$, para pinçar o músculo adutor no vértice de um ângulo imaginário. $\bigcirc$ procedimento foi realizado por meio de três aferições e, posteriormente, fez-se uma média final, a qual foi utilizada como resultado. Como não há um ponto de corte definido para pacientes oncológicos, utilizou-se o ponto de corte de EMAP de 13,4 mm para mão dominante de Bragagnolo et al. ${ }^{10}$, na qual o valor inferior ao mesmo indica má nutrição.

\section{Avaliação da Proteína C Reativa}

Para análise da PCR foi coletado $1 \mathrm{ml}$ de sangue, este colocado em um tubo BD Vacutainer (ref. 367983), em seguida direcionado para o Laboratório de Análises Clínicas do Hospital das Clínicas, na qual foram realizadas as análises pelo método Imunoturbidimétrico com látex.

\section{Análise Estatística}

Os dados foram expressos como média \pm desvio padrão. Para comparar variáveis não-paramétricas utilizou-se o teste Mann-Whitney e para variáveis paramétricas, o teste T Student. $O$ teste qui-quadrado foi utilizado para verificar a diferença entre as variáveis categóricas. A análise de regressão logística usando o modelo Crude (não ajustado) e modelo 1 (ajustado para sexo e idade), e modelo 2 (ajustado para sexo, idade, IMC) foi aplicada para avaliar o odds ratio (associação) da EMAP com PCR. Os testes estatísticos foram realizados no software MedCalc ${ }^{\circledR}$, Bélgica, e adotou-se $p<0,05$ como estatisticamente significante.

\section{RESULTADOS}

Participaram do estudo 110 pacientes oncológicos hospitalizados, destes, 55 homens e 55 mulheres. A caracterização da população está demonstrada na Tabela 1 .

Dentre os pacientes estudados, 100 apresentaram EMAP $<13,4$, sendo 48 homens e 52 mulheres. Em relação à EMAP normal $(n=10)$, a amostra foi composta na sua maioria por homens. Dentre os pacientes com a EMAP alterada, observou-se que o tipo de câncer sólido $(n=78)$ é o de maior prevalência, porém sem diferença entre os grupos.

Os pacientes com EMAP $<13,4$ também apresentaram menor peso atual (EMAP $<13,4=60,1 \pm 12,8 \mathrm{~kg}$ vs. EMAP $\geq 13,4=74,8 \pm 17,1 \mathrm{~kg}, \mathrm{p} \mathrm{0,001)}$, peso usual (EMAP $<13,4=68,3 \pm 14,1 \mathrm{~kg}$ vs. EMAP $\geq 13,4=80 \pm 14,6$ $\mathrm{kg}, \mathrm{p} 0,015)$ e IMC (EMAP $<13,4=22,7 \pm 4,7 \mathrm{~kg} / \mathrm{m}^{2} \mathrm{vs}$.
EMAP $\left.\geq 13,4=26,0 \pm 4,7 \mathrm{~kg} / \mathrm{m}^{2}, \mathrm{p} 0,041\right)$. As demais variáveis, como idade, percentual de perda de peso (\%PP), altura e PCR, não diferiram entre os grupos.

Na análise de regressão logística, a EMAP não demonstrou associação com a PCR, tanto no modelo sem ajuste (EMAP x PCR OR: 1,01; IC95\% [0,94-1,09], $p=0,65)$, quanto no modelo 1 ajustado para sexo e idade (EMAP $x$ PCR OR: 1,01; IC95\% [0,94-1,09], $p=0,61)$ e no modelo 2 ajustado para sexo, idade e IMC (EMAP x PCR OR: 1,04; IC95\% [0,96-1,13], $p=0,29$ ) (Tabela 2).

Tabela 1 - Caracterização dos pacientes segundo valores da espessura do músculo adutor do polegar (EMAP).

\begin{tabular}{lccc}
\hline \multirow{2}{*}{ Variáveis } & $(\mathrm{EMAP} \geq 13,4)$ & $($ EMAP $<13,4)$ & \\
\cline { 2 - 3 } & $\mathrm{n}=10$ & $\mathrm{n}=100$ & \\
\hline Sexo & & & \\
Masculino & 7 & 48 & $-0,18$ \\
Feminino & 3 & 52 & \\
\hline
\end{tabular}

\section{Tipo de Câncer ${ }^{\mathrm{a}}$}

\begin{tabular}{lccc}
\multicolumn{1}{c}{ Líquido } & 1 & 22 & \\
\multicolumn{1}{c}{ Sólido } & 9 & 78 & \\
\hline Idade (anos) $^{\mathrm{b}}$ & $57,4 \pm 18,6$ & $57,1 \pm 14,1$ & 0,96 \\
Peso Atual (kg) & $74,8 \pm 17,1$ & $60,1 \pm 12,8$ & $0,00^{*}$ \\
Peso Usual (kg) & $80 \pm 14,6$ & $68,3 \pm 14,1$ & $0,01^{*}$ \\
\%PPc & $5,3(3,1-20,3)$ & $9,9(15,0-44,2)$ & 0,25 \\
Altura ${ }^{\mathrm{b}}$ & $1,7 \pm 0,1$ & $1,6 \pm 0,1$ & 0,19 \\
IMC (kg/m²) $^{\mathrm{b}}$ & $26 \pm 4,7$ & $22,7 \pm 4,7$ & $0,04^{*}$ \\
EMAP $^{\mathrm{b}}$ & $17,6 \pm 4,2$ & $6,7 \pm 2,6$ & $<0,00^{*}$ \\
PCR $^{\mathrm{b}}$ & $9,7 \pm 9,3$ & $8,3 \pm 8,5$ & 0,62 \\
\hline
\end{tabular}

EMAP = espessura do músculo adutor do polegar; $\mathrm{PP}=$ perda de peso, $\mathrm{IMC}=$ índice de massa corporal; $\mathrm{PCR}=$ proteína $\mathrm{C}$ reativa.

${ }^{\star} \mathrm{p}<0,05$ foi considerado como significante.

Teste de qui quadrado; 'teste t de Student; 'teste Mann Whitney.

Tabela 2 - Regressão logística (OR, odds ratio) entre EMAP e PCR.

\begin{tabular}{lcccc}
\hline Variável & Análise & OR & $95 \% \mathbf{C l}$ & $\boldsymbol{p}$ \\
\hline EMAPa $^{\mathrm{x}}$ PCR & Sem ajuste & 1,01 & $0,94-1,09$ & 0,65 \\
EMAPa $\times$ PCR & ${\text { Modelo } 1^{\mathrm{b}}}$ & 1,01 & $0,94-1,09$ & 0,61 \\
EMAPa $\times$ PCR & Modelo $^{c}$ & 1,04 & $0,96-1,13$ & 0,29 \\
\hline
\end{tabular}

EMAP = espessura do músculo adutor do polegar, $\mathrm{PCR}=$ proteína $\mathrm{C}$ reativa. Modelo 1 = Ajustado para sexo, idade.

Modelo 2 = Ajustado para sexo, idade, índice de massa corporal.

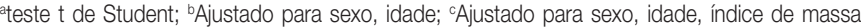
corporal (IMC). 


\section{DISCUSSÃO}

O principal achado do nosso estudo é que a EMAP não se associou com a PCR. Entretanto, até o momento, não há estudos prévios que avaliaram essa associação, sendo este um pioneiro. Outro achado do nosso estudo foi que $90 \%$ dos pacientes apresentaram EMAP alterada e menores valores de IMC, peso usual e atual. Do mesmo modo, ao avaliar pacientes paliativos com câncer, Silva et al. ${ }^{12}$ encontraram EMAP alterada em $73 \%$ dos indivíduos, considerando-os desnutridos. Estudos prévios demonstram que a EMAP apresenta correlação positiva com peso e $\mathrm{IMC}^{15,16}$. Dentre essas, o peso é o principal determinante da EMAP em uma população saudável ${ }^{15}$.

A EMAP sofre as alterações decorrentes da depleção de massa muscular, de forma similar aos demais músculos corporais. Porém, além de quantificar as mudanças na massa muscular, também possibilita o seu monitoramento. Embora não exista um ponto de corte para ser usado em pacientes oncológicos, Bragagnolo et al. ${ }^{10}$ mostraram que, em pacientes cirúrgicos, a EMAP menor que 13,4 mm é indicativo de desnutrição. Nesse sentido, a desnutrição ocasionada pela doença reduz a atividade de vida diária, acarretando na perda de massa muscular ${ }^{14,17}$.Em pacientes com câncer é comum a presença de atrofia muscular desencadeada pela própria doença, bem como pelo tratamento (quimioterápico/radioterápico) e surgimento de outras comorbidades. Tais fatores se associam a piores desfechos clínicos e exigem rápida identificação ${ }^{10}$. Além da perda de peso e da EMAP, os pacientes com câncer apresentam aumento da resposta inflamatória sistêmica de forma crônica e que pode ser facilmente usada para rastrear o prognóstico a partir da concentração sanguínea de PCR $^{18}$.

A dosagem de PCR possibilita determinar e monitorar a presença de inflamação $0^{9,19}$. Portanto, na prática clínica, o uso da PCR para monitorar a inflamação é um ótimo parâmetro, desde que sua execução seja de baixo custo.

Embora a associação entre EMAP e PCR não foi encontrada em nosso estudo, os dados possibilitam o desenvolvimento de estudos futuros que associam a EMAP a outras variáveis antropométricas e laboratoriais de fácil utilização na prática clínica, tendo em vista que a EMAP apresenta baixo custo, fácil aferição e análise que contribuem na identificação prévia de fatores ligados a piores prognósticos clínicos, como a perda muscular. Do mesmo modo, a associação com mais de um valor de PCR aferido em dias alternados pode ser mais eficaz tendo em vista que a PCR pode ser alterada por estímulos agudos?.

Dentre as limitações do estudo destacamos: i) o desenho do estudo transversal que não permite estabelecer uma relação de causa e efeito, ii) coleta de dados em um único hospital, pois não permite a generalização dos achados, e iii) a não utilização do PCR ultrassensível (PCR-us) para detecção mais precisa de quadros inflamatórios. Como pontos positivos, esse é o primeiro estudo a avaliar a EMAP como uma medida prática e simples para mensurar a massa muscular e a sua relação com a PCR. Sendo assim, no que se refere a hospitais públicos, se torna válida a utilização de métodos com custo acessível, como EMAP e PCR na identificação prévia de alterações desfavoráveis no quadro de saúde dos pacientes.

Portanto, não houve associação entre EMAP e PCR; estudos futuros no ambiente hospitalar, com pacientes com câncer, são necessários para considerar a influência de outras variáveis que possam se relacionar a EMAP e também a PCR, em especial a PCR-us, no intuito de estabelecer a relevância de métodos de baixo custo na predição de alterações desfavoráveis na muscular em pacientes oncológicos. Além disso, faz se necessário o estabelecimento de um ponto de corte para EMAP em pacientes oncológicos para que não ocorra sub ou superestimação das alterações na massa muscular.

Embora a EMAP não se associou à PCR, as análises evidenciaram a importância da continuidade de utilização desses parâmetros em demais pesquisas, tendo em vista que existem outras variáveis a serem analisadas nessa comparação. Além disso, estudos com um tamanho amostral maior seriam necessários para a determinação de um ponto de corte para EMAP sensível na avaliação de pacientes com câncer hospitalizados. Do mesmo modo, investigações futuras poderão utilizar a PCR-us ligada à maior precisão da situação inflamatória dos indivíduos hospitalizados.

\section{REFERÊNCIAS}

1. Siegel RL, Miller KD, Jemal A. Cancer statistics, 2015. CA Cancer J Clin. 2015;65(1):5-29.

2. Torre LA, Bray F, Siegel RL, Ferlay J, Lortet-Tieulent J, Jemal A. Global cancer statistics, 2012. CA Cancer J Clin. 2015;65(2):87-108.

3. Bray F, Ferlay J, Soerjomataram I, Siegel RL, Torre LA, Jemal A. Global cancer statistics 2018: GLOBOCAN estimates of incidence and mortality worldwide for 36 cancers in 185 countries. CA Cancer J Clin. 2018;68(6):394-424.

4. Siddiqui IA, Sanna V,Ahmad N, Sechi M, MukhtarH. Resveratrol nanoformulation for cancer prevention and therapy. Ann N Y Acad Sci. 2015;1348(1):20-31.

5. Teixeira BC, Lopes AL, Macedo RCO, Correa CS, Ramis TR, Ribeiro JL, et al. Inflammatory markers, endothelial function and cardiovascular risk. J Vasc Bras. 2014;13(2):108-15.

6. Shrotriya S, Walsh D, Nowacki AS, Lorton C, Aktas A, Hullihen $\mathrm{B}$, et al. Serum C-reactive protein is an important and powerful prognostic biomarker in most adult solid tumors. PLoS One. 2018;13(8):e0202555.

7. Margutti KMM, Schuch NJ, Schwanke CHA. Inflammatory markers, sarcopenia and its diagnostic criteria among the elderly: a systematic review. Rev Bras Geriatr Gerontol. 2017;20(3):441-53. 
8. Allin KH, Bojesen SE, Nordestgaard BG. Baseline C-reactive protein is associated with incident cancer and survival in patients with cancer. J Clin Oncol. 2009;27(13):2217-24.

9. Aguiar FJB, Ferreira-Júnior M, Sales MM, Cruz-Neto LM, Fonseca LAM, Sumita NM, et al. Proteína C reativa: aplicações clínicas e propostas para utilização racional. Rev Assoc Med Bras. 2013;59(1):85-92.

10. Bragagnolo R, Caporossi FS, Dock-Nascimento DB, AguilarNascimento JE. Adductor pollicis muscle thickness: a fast and reliable method for nutritional assessment in surgical patients. Rev Col Bras Cir. 2009;36(5):371-6.

11. Poziomyck AK, Corleta OC, Cavazzola LT, Weston AC, Lameu EB, Coelho LJ, et al. Adductor pollicis muscle thickness and prediction of postoperative mortality in patients with stomach cancer. Arq Bras Cir Dig. 2018;31(1):e1340.

12. Silva EHE, Borges FM, CruzFCS, Pena GG. Association between nutritional status and manual grip strength in oncologic patients in palliative care. Rev Bras Cancerol. 2018;64(4):479-87.

13. Heyward V, Stolarczyk L. Avaliação da composição corporal aplicada. Barueri: Manole; 2000. 243p.

14. Lameu EB, Gerude MF, Corrêa RC, Lima KA. Adductor pollicis muscle: a new anthropometric parameter. Rev Hosp Clin Fac Med Sao Paulo. 2004;59(2):57-62.
15. Cortez AF, Tolentino JC, Aguiar MRA, Elarrat RM, Freitas Passos RB. Association between adductor pollicis muscle thickness, anthropometric and immunological parameters in HIV-positive patients. Clin Nutr ESPEN. 2017;17:105-9.

16. Valente KP, Silva NM, Faioli AB, Barreto MA, Moraes RA, Guandalini VR. Thickness of the adductor pollicis muscle in nutritional assessment of surgical patients. Einstein (Sao Paulo). 2016;14(1):18-24.

17. Oliveira CM, Kubrusly M, Mota RS, Choukroun G, Brandão Neto J, Silva CA. Adductor pollicis muscle thickness: a promising anthropometric parameter for patients with chronic renal failure. J Ren Nutr. 2012;22(3):307-16.

18. Deans DA, Tan BH, Wigmore SJ, Ross JA, de Beaux AC, Paterson-Brown $\mathrm{S}$, et al. The influence of systemic inflammation, dietary intake and stage of disease on rate of weight loss in patients with gastro-oesophageal cancer. Br J Cancer. 2009;100(1):6309.

19. Potsch AA, Siqueira Filho AG, Tura BR, Gamarski R, Bassan R, Nogueira MV, et al. C-reactive protein diagnostic and prognostic value in patients presenting at the emergency room with chest pain. Arq Bras Cardiol. 2006;87(3):275-80.

Local de realização do estudo: Laboratório de Pesquisa em Nutrição Clínica e Esportes (Labince), Faculdade de Nutrição, Universidade Federal de Goiás, Goiânia, GO, Brasil.

Conflito de interesse: Os autores declaram não haver. 